\title{
INFLUENCE OF TEMPERATURE AND HYDROGEN ON FATIGUE FRACTURE OF 10KH15N27T3V2MR STEEL
}

\author{
Oksana Hembara, Olha Chepil \\ Karpenko Physico-Mechanical Institute, National Academy of Sciences of Ukraine and \\ Lviv Polytechnic National University, Ukraine \\ e-mail: hembara@ipm.lviv.ua \\ TARAS HEMBARA \\ Lviv State University of Life Safety, Ukraine \\ Volodymyr Mochulskyi, YaroslaV SapuzhaK \\ Karpenko Physico-Mechanical Institute, National Academy of Sciences of Ukraine
}

\begin{abstract}
A theoretical and experimental approach to prognosis of fatigue crack growth behavior and determination of the remaining resource of elements of constructions under the influence of temperature and hydrogen is discussed in the paper. Kinetic fatigue fracture diagrams of austenitic steel of 10Kh15N27T3V2MR were experimentally built and analytically described at different temperatures in a neutral environment and in hydrogen. The threshold and critical values of the stress intensity factor (SIF) were found. The durability of a turbine disk was evaluated. It is found that hydrogen reduced the remaining resource of this structural element almost by 2-3 times.
\end{abstract}

Keywords: austenitic steel, hydrogen, kinetic fatigue fracture diagrams, cyclic crack resistance, durability

\section{Introduction}

In assessing the guaranteed duration of reliable operation of turbine equipment, the concept of a park resource is used. It is based on definition of the same types of design, steel grades and conditions of operation of elements of power equipment, in which accident-free working takes place if the requirements of operating instructions are observed.

During prolonged use, various types of damage arise in turbine elements due to creep and changes in properties of the material under the influence of high temperatures, low cycle and multi-cyclic fatigue, erosive processes in the flow section, corrosion cracking under stress, hydrogen degradation, etc. But even for very long periods of operation of turbines and existing significant damage and defects, the question of extending the service life is not always uniquely solved by replacing the equipment or its parts. In many cases, further operation may be repeatedly and gradually extended, but such continuation must be preceded by a set of actions which combine a thorough inspection of the technical condition of the equipment and estimated explanation of possibilities and conditions of continuation. In determining the estimated individual resource of a turbine, the effect of all factors that determine the conditions of its further exploitation should be considered, which will allow one to make a reasonable prognosis regarding the terms of continuation of its safe and reliable exploitation.

For the solution of these questions specialists conduct more precise calculation of tensionally deformed state of elements of turbine equipment under the influence of operating loading with the use of modern calculation packages, and also prognosis of crack growth through making use approaches of linear fracture mechanics (Vasovic et al., 2014; Belogurov et al., 2004; Fishgoit 
and Kolachev, 1997; Liu and Macdonald, 1997; Jovicic et al., 2009; Kim, 1999; Panasyuk et al., 2012). By results of conducted calculations, recommendations about expedience of repair and terms of further use in the case of a sample of the crack until the next inspection are given.

The main feature of calculations for engineering metallic structures in terms of mechanics of destruction is the possibility of considering the presence of initial defects in the material or fatigue damage that arise up in the process of exploitation. Such calculations are based on research of kinetics of fatigue cracks development until they reach critical sizes, and their primary purpose is determination of the remaining resource of elements of metallic structures. In engineering constructions, the stage of development of fatigue crack can be from $10 \%$ to $90 \%$ of general durability of the product. Therefore, determination of the remaining resource of a construction acquires important practical meaning.

In diagnostics, by non-destructive testing methods, it is possible to determine the initial defect of the product (Rusyn et al., 2014; Kosarevych et al., 2016), and then to calculate the remaining durability $N$ of the element by the known (Panasyuk et al., 1994; Lu et al., 2010) formula

$$
N=\int_{l_{0}}^{l_{c}} V^{-1}(l) d l
$$

where $l_{0}, l_{c}$ are initial and critical lengths of fatigue macrocrack, accordingly, $V(l)$ is the fatigue crack growth rate (FCGR). To use formula (1.1), it is necessary to know the kinetics of growth of the crack, which is determined from kinetic equations of the fatigue crack propagation.

Many research results on fatigue failure of materials are presented in the literature (Murakami and Matsuoka, 2010; Nanninga et al., 2010; http://www.sandia.gov/matlsTechRef/; Seifert and Ritter, 2008; Rozumek et al., 2010; Toribio and Kharin, 2009; Liu and Mahadevan, 2007). But because functional purposes of structural parts used in the hydrogen environment differ, they are made of various materials and are subject to influence of hydrogen in a wide range of temperatures and pressure. There is a need to conduct experimental studies on definition of cyclic stability of cracks for metals in the hydrogen environment with various parameters. The need for such studies is also due to the fact that they would make it possible to determine general laws of destruction of materials in hydrogen in order to identify the role of one or another factor in this process. That would allow one to determine the characteristics needed to construct theoretical dependencies that may be useful for engineering calculations on determination of strength and durability of the structures under the influence of hydrogen.

In conclusion, in this article the influence of parameters of environment (temperature and pressure of hydrogen) on the process of growth of fatigue cracks in metals was experimentally investigated, and an attempt was made to construct calculation dependencies for the estimation of kinetics and determination of the period of subcritical growth of fatigue cracks in hydrogenated austenitic steel 10Kh15N27T3V2MR, which is used in aerospace engineering and energetic machine building (Belogurov et al., 2004; Fishgoit and Kolachev, 1997).

\section{Materials and methodology of researches}

We tested 10Kh15N27T3V2MR austenitic steel. Its chemical composition, mode of thermal treatment and mechanical properties at room temperature are given in Table 1.

The alloying of 10Kh15N27T3V2MR steel by titanium, aluminum and boron leads to formation of $\mathrm{TiC}$ carbides, Me3B2 borides and Ni3 (Al, Ti) intermetal ides in the total amount of 8-10\%, which significantly increases the characteristics of heat resistance and significantly affects the sensitivity to hydrogen (Balitskii et al., 2009; Balytskii et al., 2009; COST 25506-85, 1985). 
After thermal treatment according to the regime given in Table 1, grain grade 5, and particles of the intermetallic $y$-phase have a size of up to $0.40 \mu \mathrm{m}$.

Table 1. The mode of thermal treatment and mechanical properties of steel in helium (above the line) and in hydrogen under pressure of $30 \mathrm{MPa}$ after pre-hydrogenation to hydrogen content of $15 \mathrm{ppm}$ at room temperature (under the line)

\begin{tabular}{|l|c|c|c|c|c|c|c|}
\hline \multirow{2}{*}{$\begin{array}{c}\text { Chemical composition } \\
\text { mass }[\%]\end{array}$} & \multicolumn{3}{|c|}{ Thermal treatment } & \multicolumn{4}{|c|}{ Mechanical properties } \\
\cline { 2 - 8 } & Tempering & Aging & $\begin{array}{c}\sigma_{b} \\
{[\mathrm{MPa}]}\end{array}$ & $\begin{array}{c}\sigma_{0.2} \\
{[\mathrm{MPa}]}\end{array}$ & $\begin{array}{c}\delta \\
{[\%]}\end{array}$ & $\begin{array}{c}\psi \\
{[\%]}\end{array}$ & $\begin{array}{c}K_{c} \\
{[\mathrm{MPa} \sqrt{\mathrm{m}}]}\end{array}$ \\
\hline \hline $\begin{array}{l}\text { C-0.09; Si-0.6; Cr-15.2; } \\
\text { Ni-27.1; Mo-1.41; W-1.92; } \\
\text { Mn-0.04; Ti-2.85; Al-0.29; } \\
\text { B-0.02; Co-0.10 }\end{array}$ & $1373 \mathrm{~K}$, & $1023 \mathrm{~K}, 16 \mathrm{~h}$ & 1270 & 870 & 17 & 23 & 98 \\
\cline { 3 - 8 } & & $923 \mathrm{~K}, 10 \mathrm{~h}$ & 1240 & 880 & 10 & 10 & 5 \\
\hline
\end{tabular}

Short-time stretching of five-fold cylindrical specimens with a diameter of the working part of $5 \mathrm{~mm}$ was carried out in a special chamber under the pressure of hydrogen up to $30 \mathrm{MPa}$ at a speed of $0.1 \mathrm{~mm} / \mathrm{min}$. The stress intensity factor for static load $K_{c}$ was calculated in accordance with the standard (COST 25506-85, 1985). In the high-pressure chamber, in the interval of 0.4-30 MPa, rectangular compact specimens measuring $50 \times 60 \times 20 \mathrm{~mm}$ were tested under an off-center tension at a speed of $0.1 \mathrm{~mm} / \mathrm{min}$. The values of $C_{s}$ were calculated according to the Strawley-Gross formula (Brown and Srawley, 1966).

The kinetic fatigue fracture diagrams were found using three-point bending of beam specimens with an edge crack of dimensions $160 \times 40 \times 20 \mathrm{~mm}$ at the loading frequency of $20 \mathrm{~Hz}$ and a coefficient of the stress ratio $R=0.22$ by the procedure described in (Vasovic et al., 2010; Balitskii et al., 2012). The experimental research was based on a three-point bending force scheme of a beam sample with an edge crack. Conducted successive measurements were made for the crack length $l$ and the number of loading cycles $N$ (given the parameters of the load cycle and the environment) to obtain the necessary parameters. The experiment was performed in the following sequence. The workpiece was cut with size $L \times B \times t$, adopted in accordance with the standards (Gray, 1974) for the tested material. A hub of length $l$ was applied on the surface of the workpiece in the direction of the sample height, that is, in a plane perpendicular to the maximum tensile stress. The radius of curvature of the top was $\rho=0.1 \mathrm{~mm}$ (Fig. 1 ); the edges (ends) of the sample were threaded. The surface perpendicular to the notch plane was polished to the required purity, since the moment of the occurrence of a crack was fixed with the help of optical equipment. The fatigue crack was taken out in the prepared sample in the air. Its length had to satisfy the condition: $l_{1}-h=2 \mathrm{~mm}$ (where $l_{1}-$ crack length with hub).
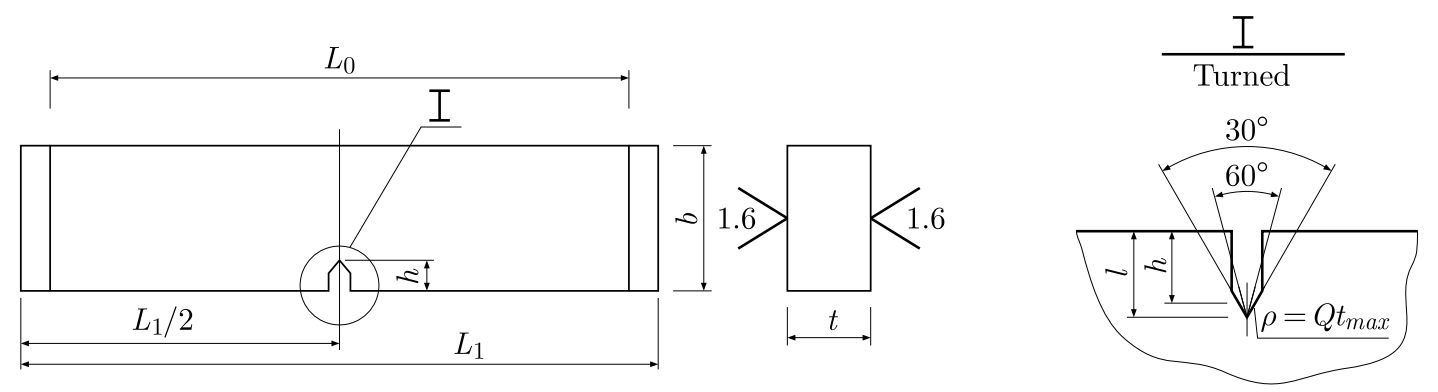

Fig. 1. Beam sample for research on cyclic crack resistance

A fatigue crack $2 \mathrm{~mm}$ long was preliminarily grown with a mechanical notch. We placed specimens with cracks in a high-pressure chamber, set on EUS-40 unit, and upon attaining required parameters of the hydrogen atmosphere, loaded them according to the known technique, 
which determination of the threshold $\Delta K_{t h}$ in hydrogen. The lengths of cracks were measured by changing the parameters of the electromagnetic vortex currents field. The method of controlling the crack length was based on the effect of a change in the electric support and a change in the voltage of the electromagnetic field of the eddy current, which was excited on the sample surface in the crack propagation region.

The field excited by the coil, incident on the not perfect metal surface, was redistributed and further changed its parameters in comparison with the field of the defect-free surface. If we assume that the crack on the surface of the sample is the main defect that causes constant redistribution of the electromagnetic field of the eddy current, then, by fixing the changes in certain parameters of this field, we can judge the growth of the crack.

The advantage of this method, in comparison with the known ones, is that the use of eddy currents allows one to transfer the spectrum of control signals to the high-frequency region using filters, which allows significant separation of the influence of the main types of interference. In addition, the use of the eddy current method allows one to increase the amplitude of the information signal by several orders of magnitude, and also significantly improves the accuracy of the crack length control and simplifies the equipment.

The scheme of the device by which this method was implemented is in (Fig. 2). It consists of two inductors, a generator, an amplifier, a filter and a recording device. Before the experiments, the inductance coils were mounted on the surface of the sample so that the top of the hub was at the same distance from their edges. The generator was connected to the first coil, called excitation coil. The amplifier, filter and recording device were connected to the second coil, called the receiver.

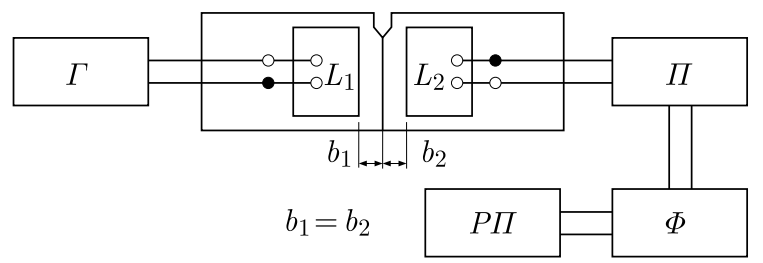

Fig. 2. Scheme of the device for measurement of length of the crack by measuring parameters of the electromagnetic field of the vortex current

In order to increase the sensitivity of the device, an amplifier was inserted in its scheme, which allowed one to increase the relative change in the value of the control signal. It should also be noted that in order to obtain correct results in determining the parameters of cyclic crack resistance in the hydrogen environment using the vortex flow method, the surface of a batch of research samples must be processed to the same purity class. In addition, the placement of the inductance coils on the sample surface should be clearly the same. Transducers were preliminarily calibrated in helium by comparing changes in the vortex currents caused by the growth of a crack, and changes in the length of cracks measured optically on a specimen with KM-8 cathetometer. We constructed kinetic fatigue fracture diagrams in helium and hydrogen under pressures up to $30 \mathrm{MPa}$ at temperatures of 293 to $673 \mathrm{~K}$ from experimentally obtained lengths of cracks $\Delta l$ during $\Delta N$ cycles under the load $F$.

The fractographic examination was performed on a scanning electron microscope Evo-40XUP with INCA Energy 350 microanalysis system. Metallographic studies were conducted with optical microscope Neofot-21, X-ray investigations were conducted on diffractometer DRON-3. The hydrogen content in the metal was determined by chromatographic analysis that was done with Leko TCH 600.

To determine the indicated mechanical characteristics in hydrogen, the working chambers were preliminarily evacuated, blown-out with hydrogen, again evacuated, and filled up with hydrogen to a given pressure. At high temperatures, the samples were kept under test conditions 
for $0.5 \mathrm{~h}$ to establish a thermal equilibrium. To compare the effect of so-called external and internal hydrogen (Balitskii et al., 2009, 2010, 2012; Balytskyi et al., 2009; Gray, 1974) as well as to determine the effect of dissolved hydrogen due to the prolonged exploitation of constructions on the properties of steels, a part of the samples was preconditioned for $10 \mathrm{~h}$ in a hydrogen atmosphere under a pressure of hydrogen of $30 \mathrm{MPa}$ and temperature $623 \mathrm{~K}$ to hydrogen concentration of about 15 ppm. It is known (Balitskii et al., 2010, 2012; Gray, 1974) that a significant deterioration of the mechanical properties of austenitic steels of the type 10Kh15N27T3V2MR in the temperature range 293-373 K occurs only after the high-temperature prehydrogenation. The tests were carried out in high pressure chambers to simulate the operating conditions of the power equipment.

\section{Analytical kinetic fatigue fracture diagrams}

The assessment of regularities in changing of size of the crack, depending on time or number of load cycles $N$ at the stage of development of macro cracks, is one of the most important tasks of the theory of fatigue fracture. In terms of linear fracture mechanics, FCGR should be determined primarily by changing the value of SIF, that is

$$
V=V\left(K_{1 \max }, K_{1 \min }, c_{i}\right) \quad i=1,2,3, \ldots
$$

where $c_{i}$ are constants of the material. Relation (3.1) reflects the fact that geometry and loading scheme are not affected by the dependence of FCGR on SIF under small scale yielding conditions, and only the material effects it, what is sufficiently well confirmed experimentally (Panasyuk et al., 1994; Lu et al., 2010; Murakami and Matsuoka, 2010; Nanninga et al., 2010; http://www.sandia.gov/matlsTechRef/; Seifert and Ritter, 2008; Rozumek et al., 2010; Toribio and Kharin, 2009; Liu and Mahadevan, 2007).

Dependencies of type (3.1) that are constructed on the basis of experimental studies are called kinetic fatigue fracture diagrams (KFFD). They reflect the most important patterns of development of fatigue cracks in the material and are a basic indicator of its resistance to cyclic cracks.

For the analysis and practical application of KFFD to materials, it must describe them analytically, that is, to present relation (3.1) in an explicit form. Recently, dozens of different variants for describing complete KFFD and its individual sites (Vasovic et al., 2014; http://www.sandia.gov/matlsTechRef/; Panasyuk et al., 2001) are offered in the literature. Among them, it is worth highlighting, first of all, the Perris formula for describing the midamplitude section of the diagram

$$
V=C K_{I \max }^{n}
$$

The value $n$ defines the angle of inclination of the straight line with respect to the abscissa axis. Between the parameters $C$ and $n$, there is a certain correlation, therefore, they can not be considered as independent values. This defect is absent in the modified Perris equation proposed in (Panasyuk et al., 1994)

$$
V=10^{-7}\left(\frac{K_{I \max }}{K_{I}}\right)^{n}
$$

Here $K_{I}$ corresponds to the amplitude value of SIF, in which the growth rate of the crack is equal to $10^{-7} \mathrm{~m} /$ cycle. A relatively simple four-parameter formula which well describes KFFD in the full range of variation of FCGR was proposed in (Vasovic et al., 2014)

$$
V^{-1}=A\left[\left(\frac{K_{t h}}{K_{f c}-K_{1 \max }}\right)^{m}-1\right]
$$


Along with these relations in the study of the kinetic fatigue fracture of structural elements, it is convenient to use the characteristic function of fatigue fracture (Panasyuk et al., 1994), which is the inversed value to FCGR

$$
\Phi(\lambda)=\frac{1}{V} \quad \lambda=1-\frac{K_{1 \max }}{K_{f c}}
$$

The representation of KFFD in the coordinates $\Phi, \lambda$ has a certain physical content - the area is limited by such a diagram and the abscissa axis is the relative value of the durability $N$ of the material, by which it is easy to determine the resource of an element of metallic structures.

In the case, when $K_{t h} \leqslant K_{1 \max } \leqslant K_{f c}$, the function $\Phi(\lambda)$ is presented as (Panasyuk et al., 1994)

$$
\Phi(\lambda)=A\left[\left(\frac{\lambda_{0}}{\lambda_{0}-\lambda}\right)^{m}-1\right]
$$

Then, taking into account relation (3.5), (3.6) for FCGR becomes

$$
V=\frac{1}{A}\left[\left(\frac{K_{f c}-K_{t h}}{K_{1 \max }-K_{t h}}\right)^{m}-1\right]^{-1}
$$

Panasyuk et al. (1994) believed that lowering the resistance of the material to fatigue crack propagation was a result of the influence of hydrogen on deformation properties of the prefracture zone. Therefore, a relation for description of the kinetics of crack propagation under the effect of a cyclic load and hydrogen diffusion into the prefracture zone has the following form

$$
V_{H}=\frac{1}{A}\left\{\left[\frac{\lambda_{0}\left(1-\lambda+\sqrt{(1-\lambda)^{2}+\alpha C_{H}}\right)}{\left(\lambda_{0}-\lambda\right)\left(1-\lambda+\sqrt{(1-\lambda)^{2}+\alpha C_{H}}+\alpha C_{H} t\right)}\right]^{m}-1\right\}^{-1}
$$

where

$$
\lambda=1-\frac{K_{1 \max }}{K_{f c}} \quad \lambda_{0}=1-\frac{K_{t h}}{K_{f c}}
$$

\section{Results and discussion}

\subsection{Influence of temperature and pressure of hydrogen on the parameters of cyclic crack} resistance and FCGR

Temperature dependencies of the threshold value $\Delta K_{t h}$ and critical SIF $K_{f c}$ of steel 10Kh15N27T3V2MR represented in Fig. 3. From the given graphs it ensures that both the threshold values SIF and critical $K_{f c}$ vary with temperature changes, as in helium so in hydrogen. With the increase of temperature, the values $\Delta K_{t h}$ and $K_{f c}$ are increasing too, i.e. KFFD moves to the right. In particular, in helium $K_{f c}$ changes from $43 \mathrm{MPa} \sqrt{\mathrm{m}}$ at $T=293 \mathrm{~K}$ to $55.92 \mathrm{MPa} \sqrt{\mathrm{m}}$ at $T=693 \mathrm{~K}$, in hydrogen, accordingly, from $37.5 \mathrm{MPa} \sqrt{\mathrm{m}}$ to $40.12 \mathrm{MPa} \sqrt{\mathrm{m}}$. A similar situation takes place with the change of $\Delta K_{t h}$.

The influence of hydrogen on the threshold value SIF is maximum at temperature $293 \mathrm{~K}$, when $K_{\text {th }}$ goes down from $6.95 \mathrm{MPa} \sqrt{\mathrm{m}}$ for the nonhydrogenated specimens to $4.95 \mathrm{MPa} \sqrt{\mathrm{m}}$ for hydrogenated specimens. In general, the effect of hydrogen on the threshold value of cyclic cracking for steel 10Kh15N27T3V2MR is much lower than the critical value. Especially strong hydrogen affects the cyclic fracture resistance at $483 \mathrm{~K}$, reducing the critical value of SIF by $31 \%$.

In general, there is no extremum of hydrogen embrittlement on the temperature curves. The difference between the $K_{f c}$ parameter in hydrogen and a neutral medium is more significant 
(a)

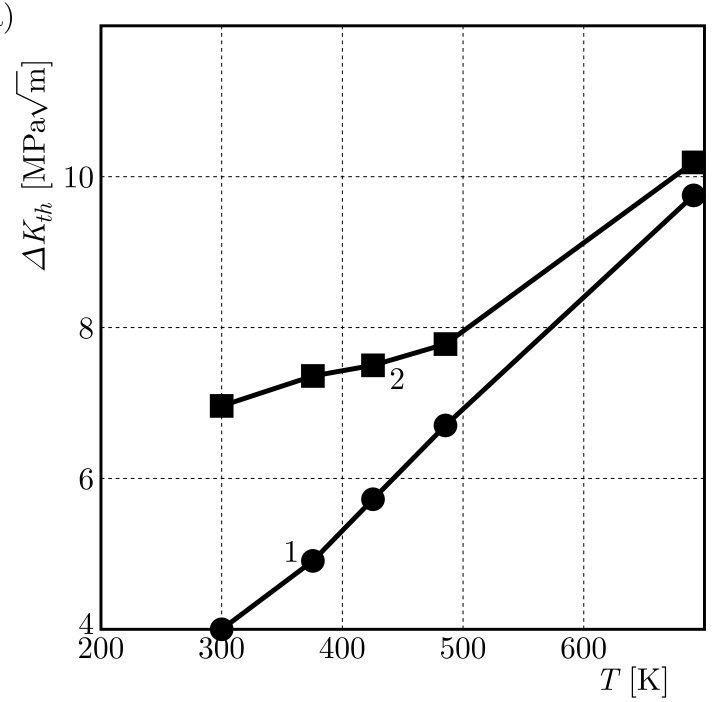

(b)

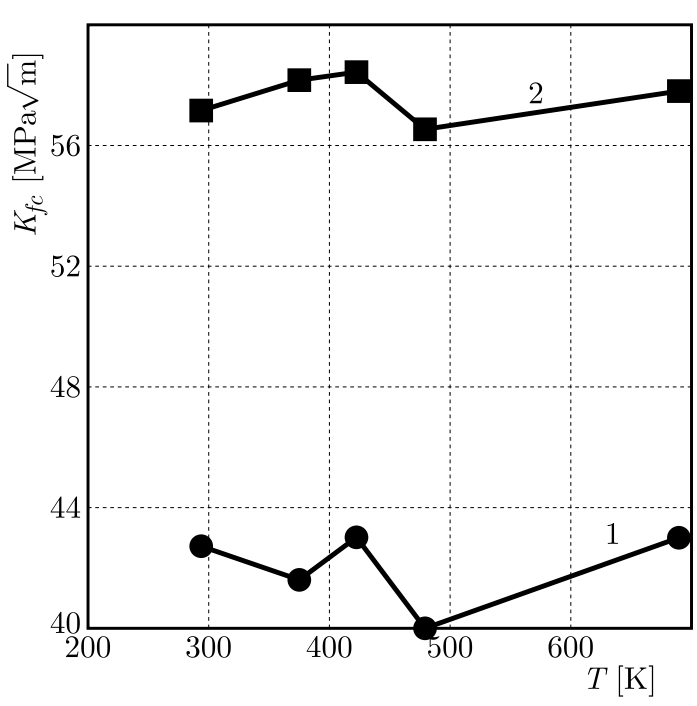

Fig. 3. Influence of temperature on the parameters of cyclic crack resistance $\Delta K_{t h}$ (a) and $K_{f c}$ (b) in helium (2) and in hydrogen (1) pressure $30 \mathrm{MPa}$ after prehydrogenation (623 K, $30 \mathrm{MPa}, \mathrm{H} 2,10 \mathrm{~h}$ )

and almost the same at all temperatures. Therefore, the negative effect of the medium on $\Delta K_{t h}$ decreases with increasing temperature.

To describe the process of fatigue fracture of metals in helium and hydrogen, it is necessary to determine the constants $A, \lambda_{0}, m, \alpha C_{H}$. To solve this problem, it is necessary to have experimental KFFD for this material, obtained in vacuum and at a certain temperature and pressure of hydrogen. At first, using a kinetic diagram built on the basis of experimental results found from the studies on samples in helium at a temperature of $293 \mathrm{~K}$ (Fig. 3a, black circles), we determined the threshold $K_{t h}$ and critical value $K_{f c}$ of the SIF that were, correspondingly, equal to $K_{t h}=4.95 \mathrm{MPa} \sqrt{\mathrm{m}}, K_{f c}=43 \mathrm{MPa} \sqrt{\mathrm{m}}$.

Then, using the formula

$$
\lambda_{0}=1-\frac{K_{t h}}{K_{f c}}
$$

we found $\lambda_{0}=0.885$.

Applying the least-squares method to the calculations, we determined values of $A=1.976 \cdot 10^{7}$ and $m=0.74$. As a result, we obtained an equation that characterizes the process of fatigue fracture of steel 10Kh15N27T3V2MR in helium at a temperature of $293 \mathrm{~K}$

$$
V=\frac{1}{1.976 \cdot 10^{7}}\left[\left(\frac{0.885}{0.885-\lambda}\right)^{0.74}-1\right]^{-1}
$$

Substituting running values in (4.3), found by the dependence

$$
\lambda=1-\frac{K_{I}}{K_{f c}}
$$

we build an analytical KFFD (Fig. 4a, curve 1). Being sure of correctness of the found $A$, $\lambda_{0}, n$, we move to definition of the component $\alpha C_{H}$ that considers the influence of hydrogen concentration on the growth rate of the fatigue crack.

To determine the value $\alpha C_{H}$, an experimental KFFD obtained for the case of the effect of a gaseous hydrogen medium on the material at a temperature of $293 \mathrm{~K}$ and a pressure of $15 \mathrm{MPa}$ was used (Fig. 4b, light circles). Repeatedly applying the least squares method to calculations, we find: $\alpha C_{H}=6.73 \cdot 10^{-4}$. Based on the found constants, equation (3.9) looks like 


$$
V=\frac{1}{1.976 \cdot 10^{7}}\left[\left(\frac{0.88338\left[1-\lambda+\sqrt{(1-\lambda)^{2}+6.73 \cdot 10^{-4}}\right]}{(0.88338-\lambda)\left[1-\lambda+\sqrt{(1-\lambda)^{2}+6.73 \cdot 10^{-4}}\right]+6.73 \cdot 10^{-4}}\right)^{0.74}-1\right]^{-1}
$$

Based on (4.4), we construct an analytical KFFD for steel 10Kh15N27T3V2MR, which considers the influence of gaseous hydrogen at a temperature of $293 \mathrm{~K}$ (Fig. 4b, curve 1).

(a)

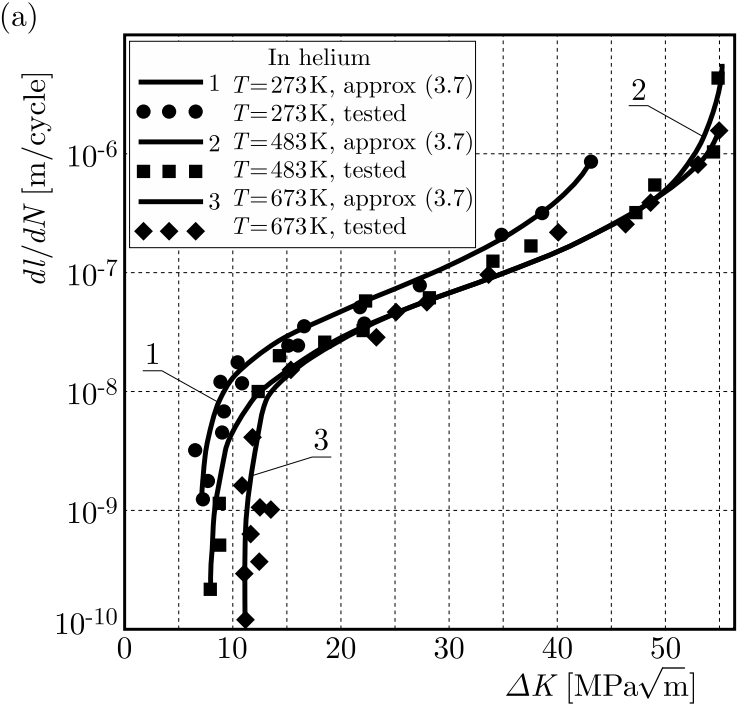

(b)

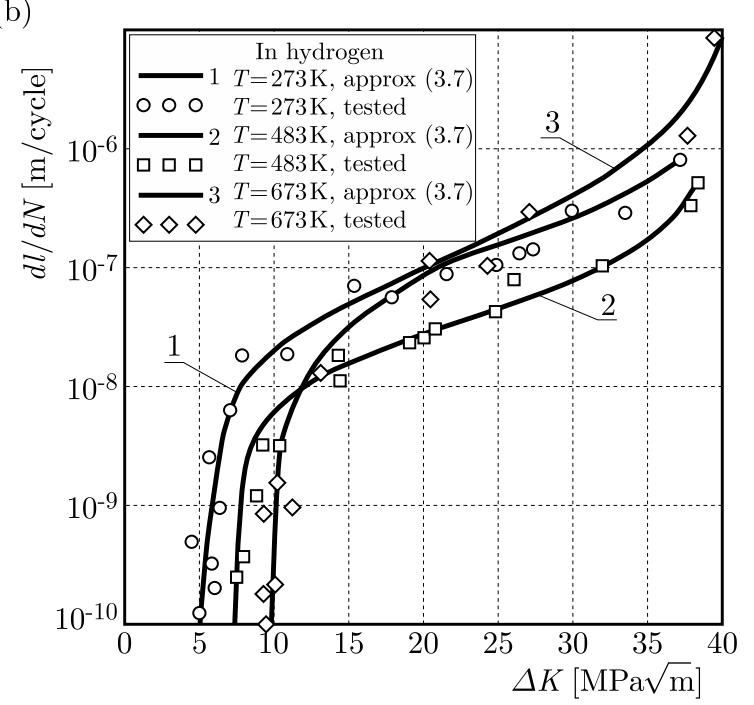

Fig. 4. Kinetic fatigue fracture diagrams of steel 10Kh15N27T3V2MR in helium (a) and in hydrogen (b) at different temperatures: $1-T=293 \mathrm{~K}, 2-T=483 \mathrm{~K}, 3-T=673 \mathrm{~K}$ (curves are approximations from formulas (3.7) and (3.8), symbols are experimental data)

Similarly, we construct an analytical KFFD in helium (Fig. 4a) and in hydrogen (Fig. 4b) at temperatures $T=483 \mathrm{~K}$ (curves 2 ) and $T=693 \mathrm{~K}$ (curves 3 ). Values of the parameters $A$, $\lambda_{0}, m, \alpha C_{H}$, threshold $K_{t h}$ and critical $K_{f c}$ are given in Table 2 .

\section{Table 2}

\begin{tabular}{|c|c|c|c|c|c|c|c|c|}
\hline $\begin{array}{c}T \\
{[\mathrm{~K}]}\end{array}$ & $\begin{array}{c}K_{t h} \\
{[\mathrm{MPa} \sqrt{\mathrm{m}}]}\end{array}$ & $\begin{array}{c}K_{f c} \\
{[\mathrm{MPa} \sqrt{\mathrm{m}}]}\end{array}$ & $\begin{array}{c}K_{t h H} \\
{[\mathrm{MPa} \sqrt{\mathrm{m}}]}\end{array}$ & $\begin{array}{c}K_{f c H} \\
{[\mathrm{MPa} \sqrt{\mathrm{m}}]}\end{array}$ & $\begin{array}{c}A \\
\mathrm{cycle} / \mathrm{m}\end{array}$ & $\begin{array}{c}m \\
{[-]}\end{array}$ & $\begin{array}{c}\alpha C_{H} \\
{[-]}\end{array}$ & $\begin{array}{c}\lambda_{0} \\
{[-]}\end{array}$ \\
\hline \hline 293 & 6.95 & 43 & 4.95 & 37.5 & $1.976 \cdot 10^{7}$ & 0.74 & $6.74 \cdot 10^{-4}$ & 0.885 \\
\hline 483 & 7.46 & 55.17 & 7.12 & 37.9 & $1.754 \cdot 10^{7}$ & 0.82 & $6.21 \cdot 10^{-4}$ & 0.865 \\
\hline 673 & 10.23 & 55.92 & 9.54 & 40.12 & $1.225 \cdot 10^{7}$ & 0.91 & $5.38 \cdot 10^{-4}$ & 0.835 \\
\hline
\end{tabular}

Consequently, the described approach can be successfully applied to predict the rate of fatigue crack propagation in materials exposed to hydrogen for any temperature, taking advantage of dependence (3.8). Correctness is confirmed by comparing the experimental results and analytically constructed curves.

However, for its implementation, it is necessary to construct at least three experimental KFFD at different temperatures in helium for the determination of constants $A, \lambda_{0}, m$, and three kinetic diagrams at the same temperatures and a certain hydrogen pressure for determination of $\alpha C_{H}$.

Substituting the found parameters into (3.8), we write the formulas for finding the growth rate of the fatigue crack in the medium of gaseous hydrogen of high pressure and temperatures. With the dependence $V=V(\Delta K)$, the residual durability of the construction element is determined by relation (1.1). 


\subsection{Influence of temperature and hydrogen pressure on fatigue strength of rotating elements of turbomachine}

The structural part of the turbine unit (Fig. 5), rotating with a frequency of 9000 turns/min in the medium of hydrogen for $10 \mathrm{MPa}$, was considered. The detail was modeled by the body of rotation (disk) with radius $R_{2}=240 \mathrm{~mm}$ and aperture $R_{1}=24 \mathrm{~mm}$. It is made of steel of 10Kh15N27T3V2MR type.

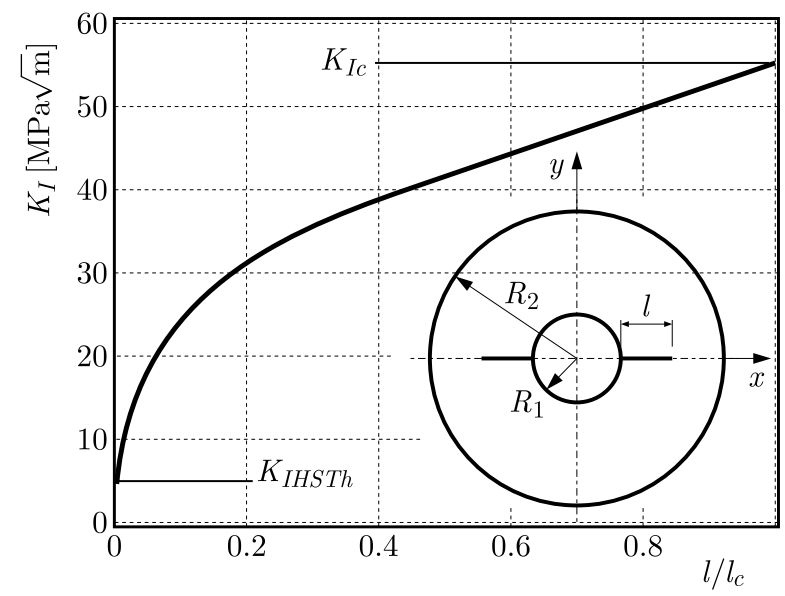

Fig. 5. The dependence of the stress intensity factor on the relative length of the crack, and a scheme of the disk with two cracks

The proposed design scheme serves for assessing the hydrogen strength of such structural elements as hydrogen cooled turbine generators, rotors and disks of gas-pumping units, turbochargers, etc.

The stress state of the body is described by the well-known solution of the theory of elasticity for rotational bodies. The tensile stresses in the diametrical section (see Fig. 5) in the condition of flat deformation are (Panasyuk et al., 2001)

$$
\sigma_{y}(x)=3.36(3+\nu) \rho R_{2}^{2} \omega^{2}\left(1+\frac{R_{1}^{2}}{R_{2}^{2}}+\frac{R_{1}^{2}}{\left(l+R_{1}\right)^{2}}-\frac{1+3 \nu}{3+\nu} \frac{\left(l+R_{1}\right)^{2}}{R_{2}^{2}}\right)
$$

where $\rho$-steel density $\left(\rho=7800 \mathrm{~kg} / \mathrm{m}^{3}\right), \nu$ - Poisson's ratio.

Calculations show that the greatest stresses arise in the region tangent to the hole, and the most vulnerable configuration of the defect in it is the presence of two starting radial cracks from the hole placed on the common diameter. For such cracks (Fig. 5) (Panasyuk et al., 2001)

$$
K_{1}=\sigma_{y}(x) \sqrt{\pi l f_{z}(l)}
$$

where the value of the function $f_{z}(l)$ is taken from a table (Murakami, 1987) and approximated by the function

$$
f_{z}(l)=1.10181+0.0475352 x^{2}-0.00148482 x^{4}
$$

The comparative analysis of the residual resource of a turbine disk with two radial cracks in different operating modes was carried out. In Fig. 6 one can see the results of calculations of the residual resource of such a disk in helium (a) and in a hydrogen-containing medium (b) at different temperatures, depending on the relative length of the crack. The number of cycles before the destruction of $N$ was calculated according to relation (1.1) using the above-stated analytical KFFD. As can be seen, a temperature change in the studied range of lengths of the cracks equally affects the residual durability (the curves are practically parallel to those 

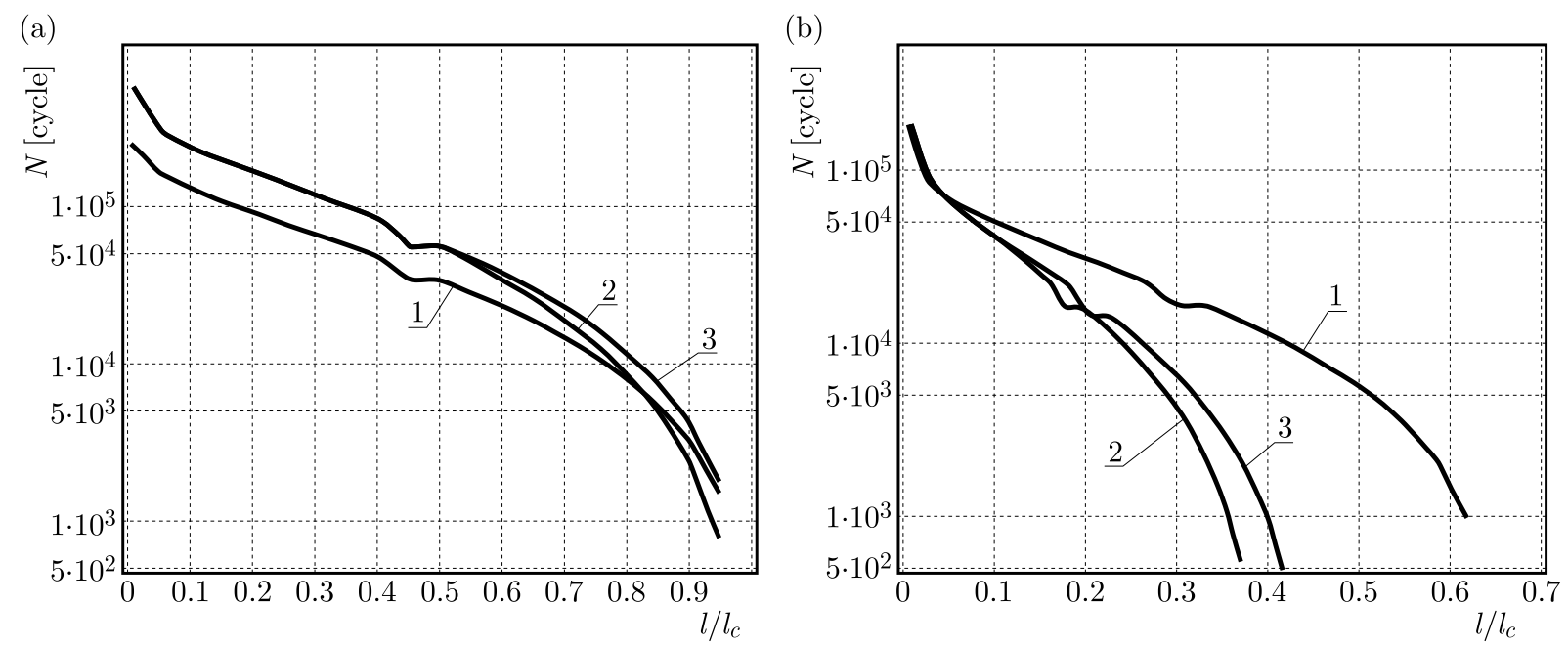

Fig. 6. Dependence of the amount of cycles to destruction from relative length of the crack in helium (a) and in hydrogen (b) for different temperatures: $1-T=293 \mathrm{~K}, 2-T=483 \mathrm{~K}, 3-T=693 \mathrm{~K}$

(a)

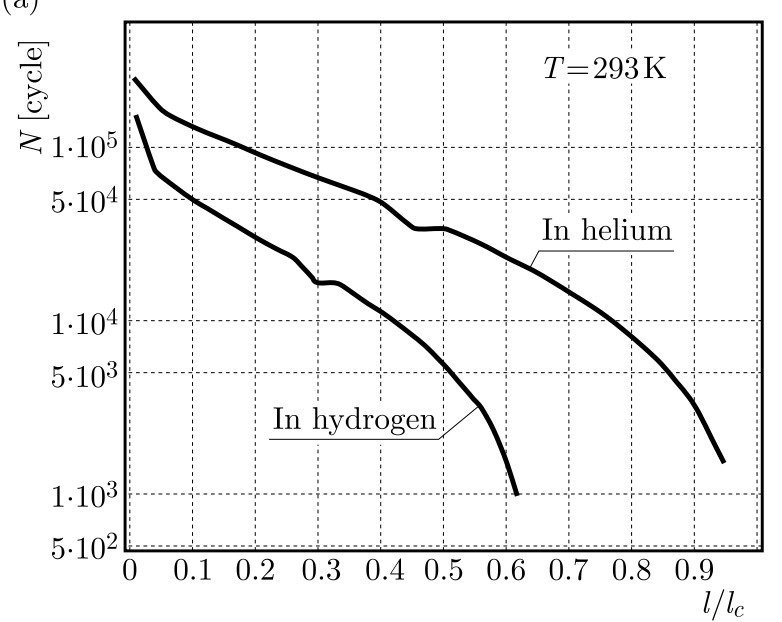

(b)

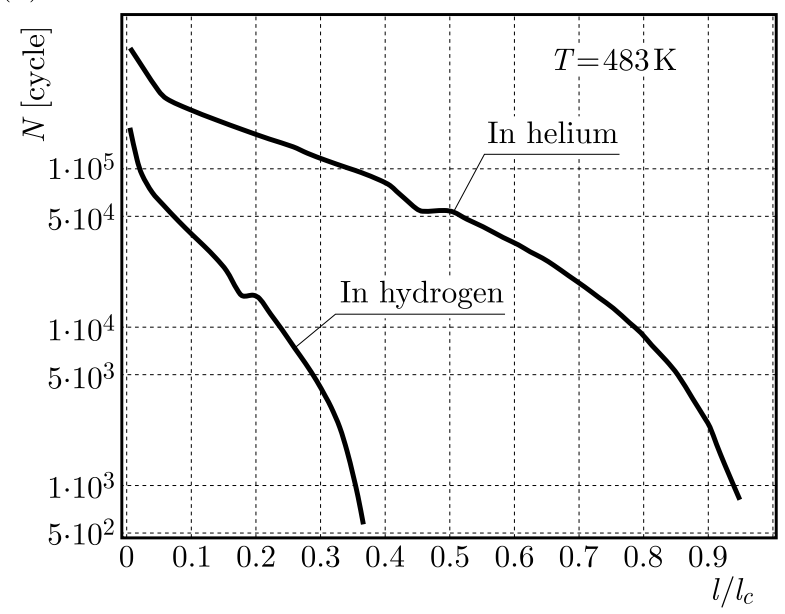

(c)

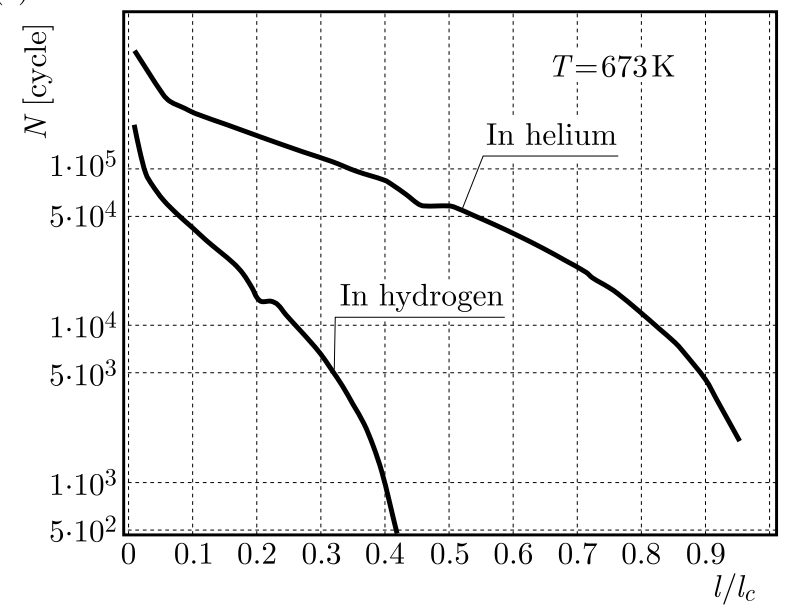

Fig. 7. Dependence of the number of cycles to fracture on relative length of the crack (in helium - solid line, in hydrogen - dashed line) for different temperatures: (a) $T=293 \mathrm{~K}$, (b) $T=483 \mathrm{~K}$, (c) $T=673 \mathrm{~K}$ 
in Fig. 6a). The situation changes in hydrogen. When increasing the crack, the resource drops significantly at higher temperatures (Fig. 6b).

In Fig. 7, the same results are given for every temperature to better demonstrate the effect of hydrogen on the remaining resource. Fracture of a disk of a turbine with two radial cracks at a ratio of $l / l_{c}=0.3$ occurs due to cracking germination in an aggressive environment for 16495 cycles, in helium - for 66421 cycles at a temperature of $293 \mathrm{~K}$ (Table 3). Hydrogen reduces the residual life at a temperature of $483 \mathrm{~K}$ at most.

Table 3

\begin{tabular}{|c|c|c|c|}
\hline$T$ & \multicolumn{2}{|r|}{$N$ [cycles] } & \multirow{2}{*}{$\begin{array}{c}l_{c} \\
{[\mathrm{~mm}]}\end{array}$} \\
\hline$[\mathrm{K}]$ & $l / l_{c}=0.3$ & $l / l_{c}=0.3$ (hydrogen) & \\
\hline 293 & $\overline{666421}$ & 16495 & $\overline{23}$ \\
\hline 483 & 119000 & 4325 & 41.9 \\
\hline 673 & 117000 & 6525 & 41.7 \\
\hline
\end{tabular}

Thus, in assessing the survivability of turbine equipment of the AES, which operates in a humidified environment, the influence of the hydrogen factor should be considered. To do this, it is necessary to have results of experimental studies of the rate of growth of the crack, depending on the SIF, considering the influence of hydrogen.

\section{Conclusions}

- The paper proposes a method for constructing experimental and analytical kinetic fatigue fracture diagrams.

- The kinetic fatigue fracture diagrams for destruction of austenitic steel 10X15H27T382MP are experimentally determined and analyzed theoretically for different temperatures in a neutral environment and in hydrogen.

- The correctness of the method is confirmed by comparing experimental results and analytically constructed curves.

- The threshold and critical values of the stress intensity coefficient are established.

- It was found that with increasing temperature from $293 \mathrm{~K}$ to $673 \mathrm{~K}$, the values of $K_{t h}$ and $K_{f c}$ increase both in helium and in hydrogen.

- Influence of hydrogen on the threshold value SIF is maximal at temperature $293 \mathrm{~K}$, when $K_{\text {th }}$ goes down from $6.95 \mathrm{MPa} \sqrt{\mathrm{m}}$ for non-hydrogenated specimens to $4.95 \mathrm{MPa} \sqrt{\mathrm{m}}$ in hydrogenated specimens.

- The effect of hydrogen on the threshold value of cyclic cracking for steel $10 \mathrm{Kh} 15 \mathrm{~N} 27 \mathrm{~T} 3 \mathrm{~V} 2 \mathrm{MR}$ is much lower than the critical value.

- Especially strong hydrogen affects the cyclic fracture resistance at $483 \mathrm{~K}$, reducing the critical value of SIF by $31 \%$.

- The residual resource of the turbine disk with two radial cracks in the air and in a hydrogen-containing environment at different temperatures, depending on the relative length of the crack, is calculated.

- The obtained results show that the presence of a relatively low concentration of hydrogen in the material can reduce durability of the mentioned elements of metal structures by 2-3 times.

\section{References}

1. Balitskit A.I., Ivaskevich L.M., Mochulskyi V.M., 2009, Temperature dependences of age-hardening austenitic steels mechanical properties in gaseous hydrogen, Proceedings on CD ROM of 
the 12th International Conference on Fracture, Ottawa, Canada, M. Elboujdaini (Edit.), T19.001, p. 7

2. Balitskit A.I., Ivaskevich L.M., Mochulskyi V.M., 2010, Crack resistance of age-hardening Fe-Ni alloys in gaseous hydrogen, 18th European Conference on Fracture. Fracture of Materials and Structures from Micro to Macro Scale, Dresden, Germany, Paper No. 80

3. Balitski A.I., Vytvytskyi V., Ivaskevich L., Eliasz J., 2012, The high- and low-cycle fatigue behavior of Ni-contain steels and Ni-alloys in high pressure hydrogen, International Journal of Fatigue, 39, 32-37

4. Balytskyi O.I., Ivaskevich L.M., Mochulskyi V.M., Holiyan O.M., 2009, Influence of hydrogen on the crack resistance of 10Kh15N27T3V2MR steel, Materials Science, 47, 2, 258-267

5. Belogurov A.I., Rachuk V.S., Rudis M.A., Sushkov A.M., Kholodnyi V.I., 2004, Strength analysis of structural elements of hydrogen power-generating equipment, Materials Science, 40, 6, $814-821$

6. Brown W.F., SRAwley J.E., 1966, Plane strain crack toughness testing of high strength metallic materials, ASTM Publications, 410

7. COST 25506-85, 1985, Methods for Mechanical Testing of Metals. Determination of the Characteristics of Crack Resistance (Fracture Toughness) under Static Loading (in Russian), Izd. Standartov, Moscow

8. Fishgoit A.V., Kolachev B.A., 1997, Strengh test in hydrogen in the aerospace industry, Materials Science, 33, 4, 568-573

9. Gray H.R., 1974, Testing for hydrogen environment embrittlement: experimental variables, [In:] Hydrogen Embrittlement Testing, ASTM STP 543, ASTM Baltimore, 133-151

10. http://www.sandia.gov/matlsTechRef/

11. Jovicic G.R., Grabulov V.K., Maksimovic S.M., Živković M.M., Jovicic N.M., Bošković G.B., Maksimovic K.S., 2009, Residual life estimation of a thermal power plant component: The high-pressure turbine housing case, Thermal Science, 13, 4, 99-106

12. KIM H.-J., 1999, Fatigue failure analysis of last stage blade in a low-pressure steam turbine, Engineering Failure Analysis, 6, 2, 93-100

13. Kosarevych R.Y., Rusyn B.P., Torska R.V., 2016, Modeling of the propagation of pitting by point processes, Materials Science, 51, 5, 673-681

14. Liu C., Macdonald D.D., 1997, Prediction of failures of low-pressure steam turbine disks, Journal of Pressure Vessel Technology, 119, 4, 393-400, DOI: 10.1115/1.2842321

15. LiU Y., Mahadevan S., 2007, Threshold stress intensity factor and crack growth rate prediction under mixed- mode loading, Engineering Fracture Mechanics, 74, 332-345, DOI: 10.1016/j.engfracmech.2006.06.003

16. Lu Z., Xiang Y., LiU Y., 2010, Crack growth-based fatigue-life prediction using an equivalent initial flaw model. Part II: Multiaxial loading, International Journal of Fatigue, 32, 376-381, DOI: 10.1016/j.ijfatigue.2009.07.013

17. Murakami Y., 1987, Stress Intensity Factors Handbook, Elsevier Science Limited, p. 1464

18. Murakami Y., Matsuoka S., 2010, Effect of hydrogen on fatigue crack growth of metals, Engineering Fracture Mechanics, 77, 1926-1940

19. Nanninga N., Slifka A., Levy Y., White C., 2010, A review of fatigue crack growth for pipeline steels exposed to hydrogen, Journal of Research of the National Institute of Standards and Technology, 115, 437-452

20. Panasyuk V.V., Andreykiv O.Y., Darchuk O.I., Kuznyak N.V., 1994, Influence of hydrogen-containing environments on fatigue crack extension resistance of metals, [In:] Handbook of Fatigue Crack Propagation in Metallic Structures, A. Carpinteri (Edit.), Amsterdam, Elsevier, $1205-1241$ 
21. Panasyuk V.V., Andreykiv O.Y., Rithie R.O., Darchuk O.I., 2001, Estimation of the effects of plasticity and resulting crack closure during small fatigue crack growth, International Journal of Fracture, 107, 99-115

22. Panasyuk V., Ivanytskyi Y, Hembara O., 2012, Assessment of hydrogen effect on fracture resistance under complex-mode loading, Engineering Fracture Mechanics, 83, 54-61

23. Rozumek D., Lachowicz C.T., Macha E., 2010, Analytical and numerical evaluation of stress intensity factor along crack paths in the cruciform specimens under out-of-phase cyclic loading, Engineering Fracture Mechanics, 77, 1808-1821

24. Rusyn B.P., Anufrieva N.P., Hrabovska N.R., Ivanyuk V.G., 2014, Nondestructive testing of the state of surfaces damage by corrosion pitting, Materials Science, 49, 4, 516-524

25. Seifert H.P., Ritter S., 2008, Corrosion fatigue crack growth behaviour of low-alloy reactor pressure vessel steels under boiling water reactor conditions, Corrosion Science, 50, 1884-1899

26. Tkachev V.I., Ivaskevich L.M., Mochulskyi V.M., 2007, Temperature dependences of mechanical properties of austenitic and martensitic steels in hydrogen, Materials Science, 45, 5, 654-666

27. Toribio J., Kharin V., 2009, Finite-deformation analysis of the crack-tip fields under cyclic loading, International Journal of Solids and Structures, 46, 1937-1952

28. Vasovic I., Maksimovic S., Maksimovic K., Stupar S., Bakic G., Maksimovic M., 2014, Determination of stress intensity factors in low pressure turbine rotor discs Mathematical Problems in Engineering, 1-9, DOI: 10.1155/2014/304638 